\title{
Colonización e infección por un microorganismo poco frecuente en una unidad de cuidados intensivos neonatales: tres recién nacidos prematuros con Elizabethkingia meningoseptica Colonization and infection with a rare microorganism in a neonatal intensive care unit: three preterm infants with Elizabethkingia meningoseptica
}

\author{
Dra. Kiymet Celik $k^{a}$ Dra. Demet Terek ${ }^{a}$, Dra. Ozgur Olukman, Profesora asociada ${ }^{a}$, Dra. Gamze Gulfidan ${ }^{b}$, \\ Dra. Sebnem Calkavur, Profesora asociada ${ }^{a}$, Dra. Ilker Devrim, Profesora asociada ${ }^{c} y$ \\ Dra. Sertac Arslanoglu, Profesora. ${ }^{a}$
}

\section{RESUMEN}

Elizabethkingia meningoseptica es un bacilo gramnegativo ampliamente distribuido en la naturaleza pero poco frecuente en humanos que se presenta en infecciones intrahospitalarias. Los avances en las instalaciones de cuidados intensivos neonatales y el uso de dispositivos médicos sofisticados fortalecen la capacidad infecciosa invasiva del microorganismo. Las manifestaciones clínicas suelen incluir bacteriemia primaria, meningitis, neumonía intrahospitalaria, bacteriemia relacionada con el uso de catéteres intravasculares einfecciones gastrointestinales y de las vías biliares. Es posible que, en entornos con recursos limitados, la falta de sistemas de diagnóstico mejorados sea una de las causas por las que no se notifican todas esas infecciones. Resulta bastante difícil distinguir entre colonización e infección, y el patrón de sensibilidad a los antibióticos es diferente. Por consiguiente, los médicos deben realizar el diagnóstico preciso para así evitar el tratamiento incorrecto. En este artículo, describimos tres casos de recién nacidos con diagnóstico de infección y colonización por E. meningoseptica con el objetivo de destacar la importancia del diagnóstico y el tratamiento oportunos de esta bacteria poco frecuente pero letal que ya está presente en las unidades de cuidados intensivos.

Palabras clave: Elizabethkingia meningoseptica, infecciones intrahospitalarias, recién nacido.

http: / / dx.doi.org/10.5546/ aap.2019.e631

Texto completo en inglés:

http: / / dx.doi.org/10.5546/ aap.2019.eng.e631

a. División de Neonatología.

b. Laboratorio de Microbiología.

c. División de Enfermedades Infecciosas Pediátricas.

Hospital Pediátrico Dr. Behcet Uz, Esmirna, Turquía.

Correspondencia:

Dra. Kiymet Celik: kiymetcelikmd@gmail.com

Financiamiento: Ninguno.

Conflicto de intereses: Ninguno que declarar.

Recibido: 24-7-2018

Aceptado: 6-6-2019
Cómo citar: Celik K, Terek D, Olukman O, Gulfidan G, et al. Colonización e infección por un microorganismo poco frecuente en una unidad de cuidados intensivos neonatales: tres recién nacidos prematuros con Elizabethkingia meningoseptica. Arch Argent Pediatr 2019;117(6):e631-e634.

\section{INTRODUCCIÓN}

El bacilo gramnegativo Elizabethkingia meningoseptica (E. meningoseptica) se encuentra ampliamente distribuido en la naturaleza, sobre todo en las plantas, en el suelo y en el agua. También puede sobrevivir en suministros de agua tratada con cloro y en el entorno hospitalario. ${ }^{1} \mathrm{Si}$ bien se trata de una bacteria de baja virulencia, puede provocar infecciones humanas poco frecuentes pero graves, especialmente en huéspedes inmunodeprimidos como los recién nacidos, a través de dispositivos permanentes colonizados, como las cánulas de intubación, los humidificadores, los circuitos de respiración y las incubadoras para recién nacidos. ${ }^{1,2}$ La evolución clínica de las infecciones por E. meningoseptica en recién nacidos suele ser grave, con un desenlace mortal o con un deterioro neurológico grave en los sobrevivientes. ${ }^{3}$ En este artículo, describimos tres casos de recién nacidos con E. meningoseptica para destacar la importancia de la evolución clínica, el tratamiento y el diagnóstico diferencial entre la infección y la colonización.

\section{CASOS CLÍNICOS \\ Caso 1}

El paciente fue un recién nacido de 26 semanas de gestación, con un peso al nacer de 1000 gramos, de sexo masculino, con una madre de 23 años de edad. El día 26 se lo derivó a nuestra unidad de cuidados intensivos neonatales (UCIN) con el diagnóstico de conducto arterial persistente (CAP) a pesar de haber recibido tratamiento con paracetamol por vía oral. Debido a la insuficiencia 
respiratoria al momento del ingreso, se lo intubó y conectó a respiración mecánica. Se le administraron dos ciclos de ibuprofeno por vía intravenosa y el CAP se cerró correctamente sin necesidad de tratamiento quirúrgico. En el día 28 de hospitalización, mientras aún estaba en ventilación mecánica, su estado clínico se deterioró, por lo que se realizaron ajustes adicionales en el respirador. Los análisis de laboratorio revelaron una concentración elevada de proteínas de la fase aguda y leucocitosis con un aumento en el recuento de neutrófilos. En la radiografía de tórax, se observaron infiltraciones pulmonares bilaterales. Se realizaron cultivos de sangre, catéter vascular, orina y líquido cefalorraquídeo (LCR). También se obtuvieron muestras de lavado broncoalveolar por sospecha de neumonía asociada al respirador. Se aisló E. meningoseptica sensible a la ciprofloxacina y la cefoperazona-sulbactam. No obstante, todos los demás cultivos fueron negativos para E. meningoseptica. Se diagnosticó neumonía asociada al respirador (NAR) de acuerdo con los criterios diagnósticos de los Centros para el Control y Prevención de Enfermedades (Centers for Disease Control and Prevention, CDC). El recién nacido respondió al tratamiento de $100 \mathrm{mg} / \mathrm{kg}$ de cefoperazona-sulbactam dos veces al día por vía venosa, administrado durante 14 días. La condición clínica mejoró gradualmente y se lo extubó al día 56 de hospitalización. En el día 91 de vida, recibió el alta tras haberse recuperado por completo.

\section{Caso 2}

El paciente fue un recién nacido de 1180 gramos, de sexo masculino, de 28 semanas de gestación, gemelo; con una madre de 33 años. Se lo derivó a nuestra UCIN en el transcurso de la primera hora de vida, tras haber sido intubado en la sala de partos. Se le administró surfactante por vía endotraqueal para el tratamiento del síndrome de dificultad respiratoria (SDR) e ibuprofeno por vía intravenosa para el CAP. Durante el seguimiento, tuvo una hemorragia intraventricular (HIV) de grado 3. Se lo extubó el día 16. No obstante, su condición clínica empeoró en el día 21 con distención abdominal y dificultad respiratoria, por lo que se lo volvió a intubar. Se diagnosticó sepsis neonatal intrahospitalaria de inicio tardío y, luego de obtener los cultivos de sangre, orina, LCR y aspirado traqueal, se inició el tratamiento empírico con vancomicina y piperacilina-tazobactam. En el cultivo de aspirado traqueal, se halló E. meningoseptica sensible a la ciprofloxacina. Dado que no se observó empeoramiento en la radiografía de tórax y que el recién nacido respondió al tratamiento antibiótico empírico, se consideró el aislamiento como colonización y no se hicieron cambios en el tratamiento. Los cultivos de aspirado traqueal de control fueron negativos para la proliferación bacteriana. Se lo extubó el día 26 y recibió el alta el día 110 con otras complicaciones debido a la prematurez.

\section{Caso 3}

La paciente fue una recién nacida de 1100 gramos, de sexo femenino, trilliza, con 27 semanas de gestación y una madre de 26 años. Se la derivó a nuestra UCIN a la quinta hora de vida con diagnóstico de SDR. Se la intubó y se le administró surfactante por vía endotraqueal. También tenía un CAP concomitante con hipotensión grave, acidosis metabólica, trombocitopenia y HIV bilateral de grado 3. Luego de completar el tratamiento para las afecciones mencionadas anteriormente, se la estabilizó y no se observaron complicaciones durante las siguientes cuatro semanas de seguimiento. Su cuadro clínico empeoró el día 30 y presentó hipotonía, apnea y acidosis mixta. Ante la sospecha de sepsis neonatal intrahospitalaria de inicio tardío, se le administró un tratamiento empírico con vancomicina, meropenem y amikacina luego de obtener los cultivos de sangre, orina y LCR. Se la intubó y conectó a un respirador de inmediato por insuficiencia respiratoria.

En el día 35, las secreciones de las vías respiratorias eran purulentas y la radiografía de tórax tenía aspecto de vidrio esmerilado, asociados con la neumonía. En los cultivos de aspirado traqueal, se observó proliferación de E. meningoseptica sensible a la ciprofloxacina y la levofloxacina. Todos los demás cultivos fueron negativos para la proliferación bacteriana. Se inició el tratamiento con ciprofloxacina. Su estado clínico se recuperó gradualmente. No obstante, durante casi dos semanas, los cultivos seriados de aspirado traqueal seguían siendo positivos para E. meningoseptica. Se interpretaron los cultivos en curso como colonización, ya que no se observó un empeoramiento en el cuadro clínico durante el seguimiento. Se la extubó el día 16 y el tratamiento con ciprofloxacina se completó a los 21 días. Fue dada de alta el día 147 con varias comorbilidades relacionadas con la prematurez. 


\section{DISCUSIÓN}

Las infecciones por bacterias gramnegativas son uno de los problemas principales de las UCIN, y se informó que las manos infectadas del personal son uno de los factores más importantes de transmisión dentro de la unidad. ${ }^{4,8}$ La mayoría de las bacterias gramnegativas sobreviven en el entorno hospitalario colonizando las paredes, las superficies de los muebles, los aparatos, los equipos médicos y otros dispositivos e instrumentos. La colonización también puede estar presente en la piel y las superficies mucosas del personal y de los pacientes. Una vez que se produce la colonización en los seres humanos, las bacterias pueden permanecer inocuas o causar infecciones invasivas, como bacteriemia primaria, NAR, bacteriemia relacionada con el uso de catéteres intravasculares o infecciones gastrointestinales. ${ }^{8}$

E. meningoseptica es un bacilo ubicuo y saprófito de transmisión hídrica que no se considera parte de la microbiota normal de los seres humanos. Tiene un nivel bajo de poder patógeno y solo un pequeño porcentaje de los pacientes colonizados contrae sepsis, especialmente aquellos que están inmunodeprimidos. ${ }^{5}$ En la bibliografía actual, se informaron epidemias intermitentes en UCIN y salas de neonatología. ${ }^{4}$ En muchos estudios ambientales, se demostró que este microorganismo puede sobrevivir en superficies húmedas como los tubos de los respiradores, los suministros de agua corriente de los hospitales, líquidos aspirados y humidificadores de oxígeno. ${ }^{2}$ La transmisión de una persona a otra no es frecuente, como lo demuestran las tasas bajas de infección en los recién nacidos. En el estudio de Gungor y cols., los investigadores afirmaron que habían aislado E. meningoseptica en líquidos de nutrición parenteral total. ${ }^{1}$

En un huésped inmunocompetente, las defensas inmunitarias eliminan rápidamente la E. meningoseptica sin necesidad de tratamiento antibiótico. No obstante, dado que la respuesta inmunitaria de los recién nacidos prematuros es débil, se trata del grupo más propenso a contraer infecciones por E. meningoseptica. ${ }^{5}$ En la serie de Issack y col., siete de ocho pacientes con meningitis neonatal por E. meningoseptica tenían un peso al nacer inferior a 2500 gramos y la edad media al momento del diagnóstico era de 10 días. ${ }^{6}$ Los cuatro pacientes de este estudio tenían $\leq 28$ semanas de gestación y pesaron menos de 1500 gramos al nacer.

En nuestra experiencia, se observaron las dos formas posibles de aislamiento bacteriano -infección y colonización- de E. meningoseptica. En el primero y en el tercer caso, la NAR fue la presentación clínica principal, diagnosticada por cultivos positivos de lavado broncoalveolar y aspirado traqueal. En el tercer caso, la evolución clínica fue bastante grave y fue necesario administrar un tratamiento con fluoroquinolonas. Por otro lado, en el segundo caso, se consideró colonización el aislamiento de E. meningoseptica en el cultivo de aspirado traqueal, ya que no había síntomas respiratorios y la radiografía de tórax era normal. En relación con la experiencia limitada que teníamos en tres de los casos, concluimos que es importante realizar diagnósticos diferenciales precisos para distinguir entre la infección y la colonización y, así, evitar tratamientos antibióticos innecesarios y costos y complicaciones relacionados con dichos tratamientos. Por lo tanto, en el segundo caso, el paciente no recibió ningún otro tratamiento que no fueran los protocolos estándar de sepsis neonatal de inicio tardío. La colonización de E. meningoseptica en los recién nacidos no siempre deriva en una infección. Además, es posible controlar los brotes de colonización adoptando las precauciones habituales. ${ }^{9}$

Se sabe que el agente es resistente a la mayoría de los antibióticos y el uso inadecuado de antibióticos empíricos podría contribuir a un desenlace clínico desfavorable. Es posible que los resultados de los antibiogramas varíen en función del método de estudio preferido. Debido a la producción de dos betalactamasas, una betalactamasa de espectro ampliado (BLEA) y una metalobetalactamasa hidrolizante de carbapenémicos, muchas cepas de E. meningoseptica suelen ser resistentes a antibióticos betalactámicos de espectro ampliado, incluidos los carbapenémicos y el aztreonam. ${ }^{7}$ E. meningoseptica también es resistente a los aminoglucósidos, al cloramfenicol y a la eritromicina. No obstante, se informó que las fluoroquinolonas, la trimetoprima-sulfametoxazol y la piperacilinatazobactam in vitro son eficaces contra este microorganismo patógeno. ${ }^{7,10}$ Observamos que las cepas aisladas en los tres casos eran resistentes a la ampicilina, la gentamicina y el meropenem, mientras que eran sensibles a la ciprofloxacina.

En conclusión, el aislamiento de E. meningoseptica en los cultivos de aspirado traqueal no siempre apunta a E. meningoseptica como la causa real de la infección. Únicamente es posible interpretarlo como colonización 
con respecto a un estado clínico favorable y resultados de laboratorio relativamente normales. Por consiguiente, en esta observación clínica, quisiéramos destacar la importancia de diferenciar la infección de la colonización para evitar tratamientos antibióticos innecesarios y complicaciones indeseables relacionadas, como también los costos elevados de los tratamientos.

\section{REFERENCIAS}

1. GüngörS, Ozen M, Akinci A, Durmaz R. A Chryseobacterium meningoseptica outbreak in a neonatal ward. Infect Control Hosp Epidemiol. 2003; 24(8):613-7.

2. Kirby JT, Sader HS, Wlash TR, Jones RN. Antimicrobial susceptibility and epidemiology of a wolrdwide collection of Chryseobacterium spp: report form the SENTRY Antimicrobial Surveillance Program (1997-2001). J Clin Microbiol. 2004; 42(1):445- 8.

3. Tai IC, Liu TP, Chen YJ, Lien RI, et al. Outbreak of Elizabethkingia meningoseptica sepsis with meningitis in a well-baby nursery. J Hosp Infect. 2017; 96(2):168- 71.

4. Hoque SN, Graham J, Kaufmann ME, Tabagchali S. Chryseobacterium (Flavobacterium) meningoseptica outbreak associate with colonization of water taps in a neonatal intensive care unit. J Hosp Infect. 2001; 47(3):188-92.

5. Bloch KC, Nadarajah R, Jacobs R. Chryseobacterium meningoseptica: An emerging pathogen among immunocompromised adults: Report of 6 cases and literature review. Medicine (Baltimore). 1997; 76(1):30- 41.

6. Issack MI, Neetoo Y. An outbreak of Elizabethkingia meningoseptica neonatal meningitis in Mauritus. J Infect Dev Ctries. 2011; 5(12):834-9.

7. Amer MZ, Bandey M, Bukhari A, Nemenqani D. Neonatal meningitis caused by Elizabethkingia meningoseptica in Saudi Arabia. J Infect Dev Ctries. 2011; 5(10):745-7.

8. Yoon HS. Two cases of Chryseobacterium meningoseptica infection in neonatal intensive care unit. Koren J Pediatr. 2007; 50(7):698-701.

9. Maraki S, Scoulica E, Manoura A, Papageorgiou N, et al. A Chryseobacterium meningoseptica colonization outbreak in a neonatal intensive care unit. Eur J Clin Microbiol Infect Dis. 2009; 28(12):1415-9.

10. Mirza HC, TuncerÖ, ÖlmezS, Şener B, et al. Clinical Strains of Chryseobacterium and Elizabethkingia spp. Isolated from Pediatric Patients in a University Hospital: Performance of MALDI-TOF MS-Based Identification, Antimicrobial Susceptibilities, and Baseline Patient Characteristics. Microb Drug Resist. 2018; 24(6):816-21. 